\title{
Optical Detection of Magnetic Resonance in Nitrogen Vacancy Centre Ensembles in Bulk Diamond using an Off-Resonant Probe Laser Beam
}

\author{
C D Macrae ${ }^{1,2}$, E Fraczek $^{3}$, M E Newton ${ }^{4}$, H Dhillon $^{5}$, A Bennett $^{5}$, M Markham $^{5}$, P Diggle $^{4}$, B G Breeze $^{4}$, M \\ Dale $^{4}$, V Savitski ${ }^{3}$, P F Griffin ${ }^{2}$, A Kemp $^{3}$, E Riis $^{2}$, G McConnell $^{1}$ \\ 1. Centre for Biophotonics, Strathclyde Institute of Pharmacy and Biomedical Sciences, University of Strathclyde, 161 Cathedral Street, \\ Glasgow, G4 ORE, UK \\ 2. Experimental Quantum Optics and Photonics, Department of Physics, University of Strathclyde, Glasgow G4 ONG, UK \\ 3. Institute of Photonics, Department of Physics, University of Strathclyde, Technology \& Innovation Centre, Level 5, 99 George Street, \\ Glasgow, G1 1RD, UK \\ 4. Department of Physics, University of Warwick, Coventry, CV4 7AL, UK \\ 5. Element Six Ltd., Global Innovation Centre, Harwell Campus, Oxfordshire, UK
}

\begin{abstract}
Nitrogen vacancy (NV) centres are a type of defect in diamond that exhibit a number of interesting properties. NV centres can be optically excited around 450-650 nm and emit fluorescence at around 550-800 nm [1]. The optical excitation also spin polarises the negatively charged NV centres, permitting optical detection of magnetic resonance [2]. This allows nanotesla scale sensitive magnetometry to be performed with nanometre scale spatial resolution [3].
\end{abstract}

Generally, fluorescence based detection is used in magnetometric applications where photons are emitted in all directions thus making collection difficult. By utilising stimulated emission, the photons could be predominantly channelled into a single mode, thus greatly simplifying collection. This is especially important as the magnetometric sensitivity is proportional to the number of collected photons [3].

To date, a direct measurement of stimulated emission from ensembles of NV centres in diamond has yet to be demonstrated experimentally. Despite this, stimulated emission depletion microscopy has been utilised in superresolution imaging that relies on the stimulated emission of single NV centres to quench fluorescence [4]. Recently, there has also been similar indirect experimental evidence of stimulated emission in NV ensembles [5].

We thus aimed to show both optical gain of a probe beam in a pumped NV-rich diamond sample and a modulation of this gain via microwave excitation of its ground level spin transitions.

The experimental set-up consisted of a pump (532 nm) and probe (723-950 nm) laser beam combined with a dichroic mirror and focused into a bulk diamond specimen to a spot-size of around 90 microns. Pump excitation powers of up to $3 \mathrm{~W}$ and probe powers up to $0.5 \mathrm{~W}$ were used. The beams were then collimated and separated with a prism and the probe power measured with a photodiode. The diamond specimen was placed on an antenna to allow simultaneous excitation with microwaves. A double modulation scheme was employed to measure either the change in probe power due to the pump laser or the microwave excitation during any one experiment.

Depending on the diamond specimen and power used, either a relative increase or decrease in the probe beam power could be demonstrated when the pump beam was on. Regardless of whether the probe beam showed an increase or decrease overall, the probe power could be further decreased by exciting spin transitions in the NV centres using microwaves. Furthermore, this reduction in probe beam power with microwave excitation could be demonstrated using a probe wavelength of $950 \mathrm{~nm}$ where zero stimulated emission would be expected. The exact mechanism of this off-resonant detection of magnetic resonance is still under investigation.

\section{References}

[1] K. Beha, A. Batalov, N. B. Manson, R. Bratschitsch, and A. Leitenstorfer, "Optimum Photoluminescence Excitation and Recharging Cycle of Single Nitrogen-Vacancy Centers in Ultrapure Diamond”, Phys. Rev. Lett. 109, 97404 (2012)

[2] F. Jelezko, and J. Wrachtrup, "Single defect centres in diamond: A review", Phys. Stat. Sol. (a), 203, 3207-3225 (2006)

[3] A. Angerer, T. Nöbauer, G. Wachter, M. Markham, A. Stacey, J. Majer, J. Schmiedmayer, and M. Trupke, "Subnanotesla quantuminterference magnetometry with a single spin in diamond", arXiv:1509.01637 [quant-ph] (2015)

[4] S Arroyo-Camejo, M Adam, M Besbes, J Hugonin, V Jacques, J Greffet, J Roch, S W Hell, and F Treussart, "Stimulated Emission Depletion Microscopy Resolves Individual Nitrogen Vacancy Centers in Diamond Nanocrystals", ACS Nano, 7, 10912-9 (2013) [5] J Jeske, D W M Lau, L P McGuinness, P Reineck, B C Johnson, J C McCallum, F Jelezko, T Volz, J H Cole, B C Gibson, A D Greentree. "Stimulated emission from NV centres in diamond", arXiv:1602.07418 [quant-ph] (2016) 